\title{
Design of Focused Ultrasound Array for Non-Invasive Transesophageal Cardiac Ablation
}

\author{
Hotaik Lee*,1, David Francischelli², and Nadine Barrie Smith ${ }^{3}$ \\ ${ }^{1}$ LIG NEX1 Co., Ltd., R\&D Center, Yongin, Gyeonggi, Rep. of Korea \\ ${ }^{2}$ Medtronic, Inc., Atrial Fibrillation Technologies, Minneapolis, MN 55428, USA \\ ${ }^{3}$ Graduate Program in Acoustics, The Pennsylvania State University, University Park, PA 16802, USA
}

\begin{abstract}
Atrial fibrillation (AF) is the most common type of arrhythmia, affecting more than 2.2 million Americans. One effective treatment is cardiac ablation, which shows a high rate of success in treating paroxysmal AF. As a prevailing modality for this treatment, catheter ablation using radiofrequency has been effective, but comes with measurable morbidity and significant costs and time associated with this procedure for permanent or persistent AF. To address these issues, an ultrasound applicator for cardiac ablation without surgical incisions or blood contact was designed, developed and evaluated in this study. To initially design a transesophageal applicator using therapeutic ultrasound capable of creating atrial lesions from the esophagus, sound pressure fields in a simple tissue model were numerically determined. Based on the simulation results for transducer arrays together with current transesophageal medical devices and the throat anatomy, we designed, fabricated, and tested a focused ultrasound applicator that can be inserted into the esophagus for noninvasive cardiac ablation. The ultimate goal in this project is to bring this applicator as close as possible to the heart to effectively deliver ultrasound energy and create electrically isolating lesions in myocardial tissue, which replicate the currently used Maze procedure. The transducer design is a two-dimensional sparse phased array with flat tapered elements operating at 1.6 MHz. This array uses 64 active elements spatially sampled from 195 rectangular elements. Its probe head housing is $19 \mathrm{~mm}$ in diameter and incorporates an acoustic window. A prototype applicator has been successfully tested in vitro using fresh porcine myocardial tissue. The results demonstrated a potential applicability of an ultrasound applicator to transesophageal cardiac surgery in AF treatment.
\end{abstract}

Keywords: focused ultrasound, transesophageal ablation, atrial fibrillation, sparse array.

\section{INTRODUCTION}

Atrial fibrillation (AF) is the most common type of arrhythmia, affecting more than 2.2 million Americans [1,2]. The prevalence of AF increases with age (9\% of people older than 80 years) and it can lead to serious complications such as a stroke or a pulmonary embolism [3,4]. One effective treatment is cardiac ablation, which shows a high rate of success $(70 \%$ or more for paroxysmal $\mathrm{AF}$ and $50 \%$ or more for persistent $\mathrm{AF}$ ) in treating paroxysmal $\mathrm{AF}$ [5]. As a prevailing modality for this treatment, catheter ablation using radiofrequency has been effective, but there is measurable morbidity and significant costs and time associated with this procedure for permanent or persistent AF [6]. The cost of the catheter ablation is reported ranging from $\$ 16,278$ to $\$ 21,294$ [7] and the recurrence of AF is approximately $40 \sim 50 \%$ following the procedure [5]. To address these issues, demands from physicians for non-invasive cardiac ablation have increased [8]. A non-invasive treatment damages the targeted areas of tissue without affecting surrounding tissues or blood vessels, and may permit treatment of AF without surgical incisions in an operating room, which should translate into reducing the risk and disadvantages in invasive methods. Consequently, its morbidity would be

*Address correspondence to this author at the 148-1 Mabuk Giheung Yongin Gyeonggi, Korea / LIG Nex1, Korea; Tel: 82-31-288-9523; Fax: 8231-288-9208; E-mails: hotaik@gmail.com; htlee6810@ hotmail.com expected to be decreased due to reduced secondary complications from the invasive procedure such as infections. It can also lead to shorter recovery time compared to long hospitals stays from surgery of opening the chest, with corresponding reduced costs.

Ultrasound energy has gained interest for clinical application for decades due to its non-invasive characteristics. Since the early 1940 s, the therapeutic use of high intensity focused ultrasound (HIFU) to destroy tissue has been inves tigated [9-11]. Specifically for cardiac tissue, surgical studies using catheter-based ultrasound transducers to treat arrhythmias have been reported $[12,13]$. Because ultrasound phased arrays have the advantage of electronically moving the focal point and modifying the focal area without mechanical steering, they have been extensively studied since the 1990s to evaluate the feasibility of HIFU surgical applications [1416]. In addition, recent feasibility studies indicate that it is possible for HIFU to include intracavitary treatment as a non-invasive alternative to open surgery $[17,18]$.

The purpose of this study was to evaluate the feasibility of transesophageal cardiac surgery in atrial fibrillation treatment using therapeutic ultrasound energy without surgical incisions or blood contact. In this paper, the design, development, and evaluation of an ultrasound applicator capable of creating thermal lesions in the myocardium from the esophagus are presented. Because the esophagus is close to the posterior of the left atrium (only a few millimeters be- 


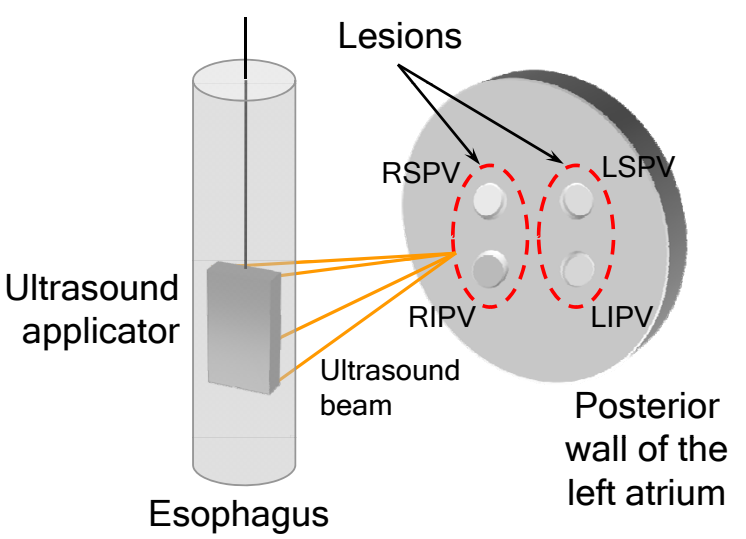

Fig. (1). Schematic of the non-invasive transesophageal cardiac surgery using ultrasound (R: Right; L: Left; S: Superior; I: Inferior; PV: Pulmonary Vein).

tween them), this position makes it attractive for incisionless surgery on selected areas of the heart. The ultimate goal is to bring an applicator as close as possible to the heart to effectively deliver ultrasound energy and create electrically isolating lesions in myocardial tissue (Fig. 1) through replication of the Maze procedure currently in use [19,20]. The Maze procedure is a surgical operation that treats $\mathrm{AF}$ by creating a grid of incisions resulting in nonconductive scar tissue in the atria [21]. Tactical arrangement of incisions interrupts the generation and spread of abnormal electrical impulses causing the arrhythmia. As a first step towards this goal, this research aims to design and develop a transesophageal ultrasonic probe for performing in vitro thermal ablation in the cardiac tissue.

\section{MATERIALS AND METHODS}

\subsection{Acoustic Pressure Calculations}

To initially design an ultrasound transducer array, ultrasound pressure fields in a simple tissue model were numerically determined. To calculate the pressure fields, simulation programs using the Rayleigh-Sommerfeld integral were written with MATLAB ${ }^{\circledR}$ (MathWorks, Inc., Natick, MA, USA) [22]. Acoustic pressure calculations are required to determine the size and number of array elements and to predict the ultrasound beam fields and temperature distribution produced by the applicator. The Rayleigh-Sommerfeld integral gives the pressure field produced in a medium at $x$ from a planar piston source [23]:

$$
p(\vec{x}, \omega)=\frac{j \omega \rho U_{0}}{2 \pi} \int_{S} \frac{\exp (-j k r)}{r} d S,
$$

where $\omega$ is the angular frequency, $\rho$ is the density of the medium, $U_{0}$ is the particle speed on the aperture surface, $k$ is the wave number, $r$ is the distance from a point on the aperture surface to field point $\vec{x}$, and $d S$ is a surface element on the surface $\mathrm{S}$.

Based on the single element beam model, the focused ultrasound field radiated from the phased array is predicted by superposition of the pressure field of each element with appropriate phase delays of driving signals. The phase of $i^{\text {th }}$ element, $\phi_{i}$ is given by:

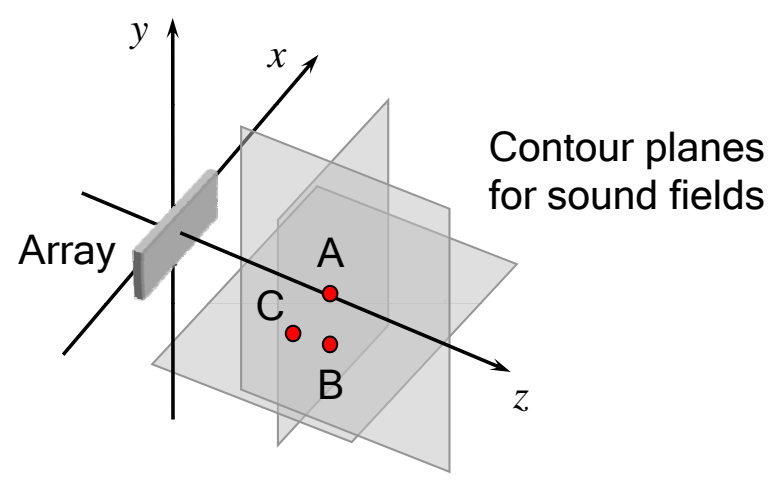

A: On-axis focal point, $[0,0,30] \mathrm{mm}$

B: Off-axis focal point, $[0,-5,30] \mathrm{mm}$

C: Off-axis focal point, $[-5,0,30] \mathrm{mm}$

Fig. (2). Position of the array and the focal points for sound fields calculations.

Table 1. Relevant Physical (Acoustical) Parameters of the Medium (Water) Used in the Sound Field Calculations

\begin{tabular}{|c|c|}
\hline Parameter & Value \\
\hline \hline Density, $\rho$ & $1000 \mathrm{~kg} / \mathrm{m}^{3}$ \\
\hline Speed of sound, c & $1480 \mathrm{~m} / \mathrm{s}$ \\
\hline Attenuation, & $0.23 \mathrm{~dB} / \mathrm{cm} / \mathrm{MHz}$ \\
\hline
\end{tabular}

$$
\phi_{i}=\frac{2 \pi}{\lambda}\left(d_{i}-d_{0}\right),
$$

where $\lambda$ is the wavelength in the interrogated medium, $d_{i}$ is the distance from the center of $i^{t h}$ element to the focal point, and $d_{0}$ is the distance from the center of the array to the focal point. Therefore, the total acoustic pressure at any field point, $\vec{x}$, produced by an array with $n$ number of elements can be written as:

$$
p(\vec{x}, \omega)=\sum_{i=1}^{n} \frac{j \omega \rho U_{0}}{2 \pi} \int_{S_{i}} \frac{\exp \left[-j\left(k r_{i}-\phi_{i}\right)\right]}{r_{i}} d S_{i} .
$$

Because of its simplicity, the Rayleigh-Sommerfeld approach has been used fairly extensively to treat the problem of ultrasound transducer designs. It can calculate ultrasound beam patterns propagated through an interface from the phased array. To quantitatively evaluate the focusing, the focal zone is generally define by the half-maximum intensity $(-3 \mathrm{~dB})$ contour. Fig. (2) shows the position of the array and the focal points for sound field calculations. The relevant physical (acoustical) parameters used in the calculations are given in Table $\mathbf{1}$.

\subsection{Design of Two-Dimensional Phased Ultrasound Array}

A transesophageal ultrasound applicator must be small enough to allow insertion into the esophagus and to avoid 


\section{Black=Active, Gray=Nonactive}

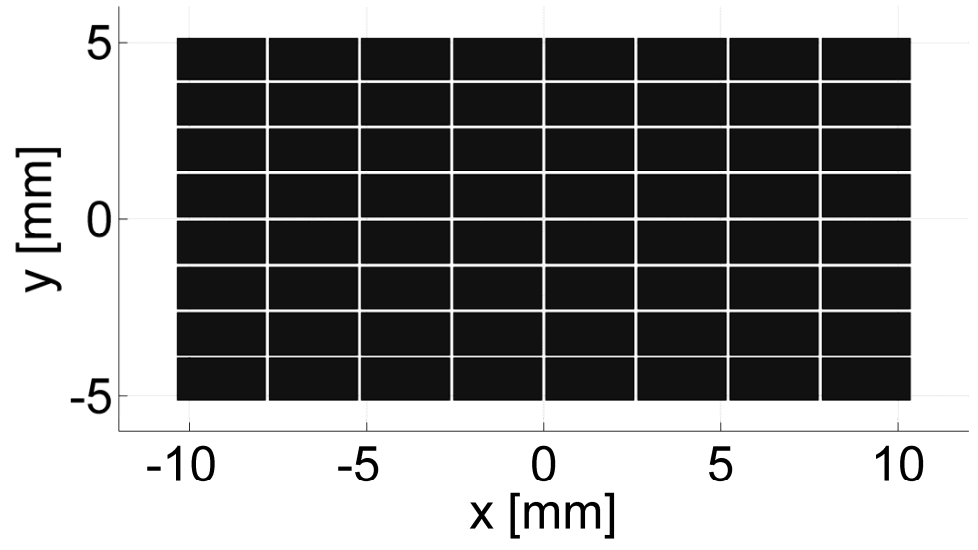

Fig. (3). Layout of the linear phased array having 64 elements $(8 \times 8)$ and a $20.7 \times 10.2 \mathrm{~mm}^{2}$ aperture size. In this set-up, all the elements are active.

\section{Black=Active, Gray=Nonactive}

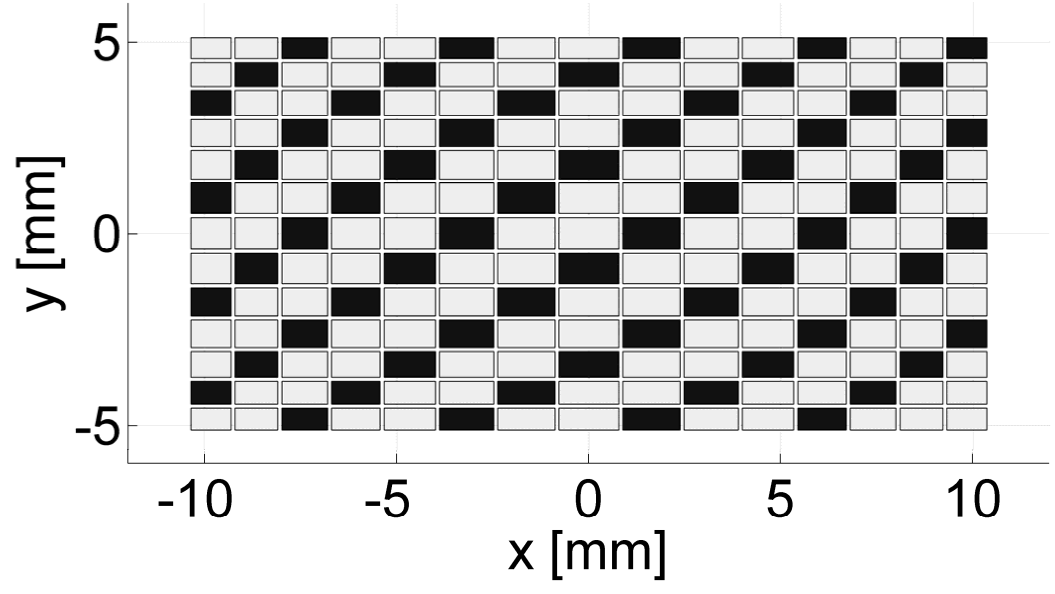

Fig. (4). Layout of the quasi-periodic sparse phased array. Active elements (black boxes) are periodically distributed within the $15 \times 13$ array $\left(20.7 \times 10.2 \mathrm{~mm}^{2}\right)$.

possible damage to the esophagus while being inserted. Also, the applicator must be capable of generating sufficient high sound intensity and of beam steering. For the design of the transesophageal ultrasound transducer, various layouts of transducer arrays were modeled and the corresponding ultrasound pressure fields were calculated. The aperture size for array design was $20.7 \mathrm{~mm} \times 10.2 \mathrm{~mm}$, which is based on the dimensions of the human esophagus and throat anatomy. According to a clinical study, the mean diameter of the esophagus is $18.6 \pm 1.6 \mathrm{~mm}$ (range 15.4-22.0) [24].

\subsubsection{Linear Phased Array}

First, the design of a two-dimensional linear phased array for focused ultrasound transducer, which has 64-elements (8 $\times 8$ ), was modeled (Fig. 3). A linear phased array with identically spaced elements is easiest to analyze and forms the basis for most array designs. The equally-sized elements operating at a frequency of $1.6 \mathrm{MHz}$ (wavelength= 1 $\mathrm{mm}$ ) were $2.5 \mathrm{~mm} \times 1.2 \mathrm{~mm}$ and they were spaced with kerf size of $105 \mu \mathrm{m}$. Anatomical information about the esophagus and the economical aspects of the producing the array were considered in determining the specifications of the model. sidered in determining the specifications of the model. This array was designed in miniature to enable its insertion into the esophagus for cardiac ablation.

\subsubsection{Quasi-Periodic Sparse Phased Array}

To improve the grating lobe problem with the linear phased array with a compact structure, a new design of the linear phased array with small pitch was suggested by increasing the number of elements in the aperture size used in the previous design. A linear phased array, which has 195elements $(15 \times 13)$ in same size, was modeled, and the sound fields for on-axis focusing and off-axis focusing were calculated (results are not shown in this paper). However, each of these elements should be connected to a channel of the ultrasound driving system. Using existing technologies, the fabrication and implementation of arrays with that large a number of elements is very difficult and expensive. A sparse array can benefit from a reduced number of elements compared with a full matrix array. Fig. (4) shows the layout of the quasi-periodic sparse phased array. Active elements (black boxes) are periodically distributed within the $15 \times 13$ array. 


\section{Black=Active, Gray=Nonactive}

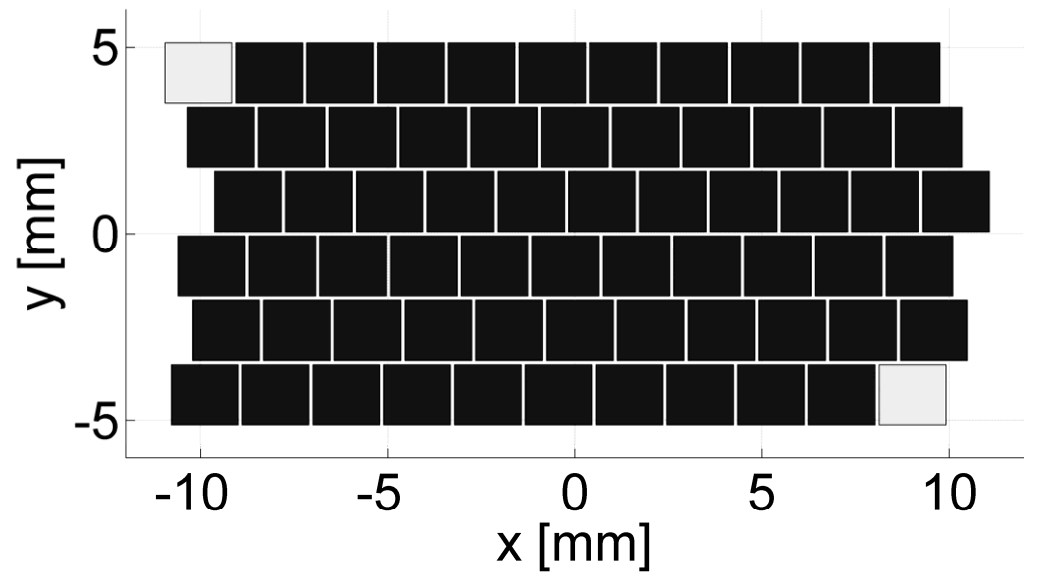

Fig. (5). Layout of the random sparse phased array. Active elements (black boxes) are randomly arranged in the $y$-direction (random offset of the element in only the $x$-direction) within the $11 \times 6$ array $\left(20.7 \times 10.2 \mathrm{~mm}^{2}\right)$.

The array has the same aperture size as the design in Fig. (3), but smaller average pitch sizes, $1.3 \mathrm{~mm}$ wide and $0.7 \mathrm{~mm}$ high. The layout involves tapered elements, ranging from 1.04 to $1.55 \mathrm{~mm}$ wide and from 0.57 to $0.84 \mathrm{~mm}$ high, according to a weighting function (a Hamming window) [25]. Ultrasound fields in a simple tissue model generated by the quasi-periodic sparse phased array were numerically examined for an on- and off- axis focusing.

\subsubsection{Random Sparse Phased Array}

One alternative design is the random sparse phased array, which is intended to control grating lobes by eliminating the periodicity in sparse arrays as well as improve efficiency of the ultrasound ablation by increasing the size of elements. Designs with larger elements for improvement of both the power transfer and acoustical efficiency were examined. The sparse array with randomly distributed elements can simultaneously enhance focusing and reduce grating lobes. Note that the meaning of "sparse" for the random sparse array is little bit different with that used for the quasi-periodic sparse array in this paper. For quasi-periodic sparse phased array, it was used to describe the thinly sampled elements instead of full matrix of the elements as shown in Fig. (4). However, for the random sparse array, sparse refers to the larger pitch size than $\lambda / 2[14]$.

The random sparse array shown in Fig. (5), which has 64 active elements among 66 rectangular elements $(11 \times 6$, two elements are surplus and inactivated), was designed based on the results of the numerical simulation using random variables (positions of geometrical centerlines of elements in rows). The dimensions of each element are $1.8 \mathrm{~mm}$ in the $x$ direction and $1.6 \mathrm{~mm}$ in the $y$-direction, which are both greater than the wavelength of $0.94 \mathrm{~mm}$. In order to furnish the array with randomness, 11 elements were aligned and equally spaced in a row ( $x$-direction), and then geometrical centerlines of 6 rows with 11 elements were randomly arranged in the $y$-direction. The random offset of the element in only the $x$-direction was achieved with the mean of 0.94 $\mathrm{mm}$ deviated from zero in the $x$-direction and the standard deviation of $0.55 \mathrm{~mm}$ for final design of the two-dimensional array with the same aperture size.

\subsection{Fabrication of the Transducer Array}

Based on the simulation results of the ultrasound field from the transducer array and its constructability, a quasiperiodic sparse phased array prototype was designed and built in-house. To handle the large electrical power needed for tissue ablation, a lead zirconate titanate ceramic (PZT-8, TRS Ceramics, State College, PA, USA) was diced into a $20.7 \mathrm{~mm} \times 10.2 \mathrm{~mm}$ segment and lapped to a thickness of $1.4 \mathrm{~mm}$, which corresponds with the resonance frequency of 1.6 MHz. The ceramic was completely diced through its thickness to form an array with a kerf of $105 \mu \mathrm{m}$ using a dicing saw (Model 982-6, Kulick and Soffa Industries, Willow Grove, PA, USA). Fig. 6(a) shows the ceramic diced into $195(15 \times 13)$ elements, including 64 active elements. The electrode surfaces on the ceramic were coated with chromium and gold sputtering. To ensure the required maximum power transfer from each array element to the ablation target, an acoustic impedance matching layer with a quarterwavelength thickness was constructed. Electrical matching circuits using inductors (L) and capacitors $(\mathrm{C})$ were constructed for impedance matching to $50 \Omega L 0^{\circ}$ between the amplifier driving system (Advanced Surgical Systems, Inc., Tucson, AZ, USA) and each transducer element. The magnetic resonance-compatible probe head housing is $19 \mathrm{~mm}$ in diameter and incorporates an acoustic window for the housing to ensure delivery of maximum acoustical power from the transducers to the ablation targets. Fig. 6(b) shows the prototype array embedded in a housing with cables and water circulation tubes. The water circulation system was designed to reduce heating from the ceramic and the high temperature in the acoustic near field to relieve unwanted heating of the esophagus and the surrounding tissues.

\subsection{Experimental Tests}

\subsubsection{Exposimetry Systems}

Ultrasonic exposimetry was performed to determine the focusing and steering characteristics of the array [26, 27]. To determine the acoustic field generated by the phased array prototype, an exposimetry system was equipped with an automated computer-controlled scanning system (Velmex 
a

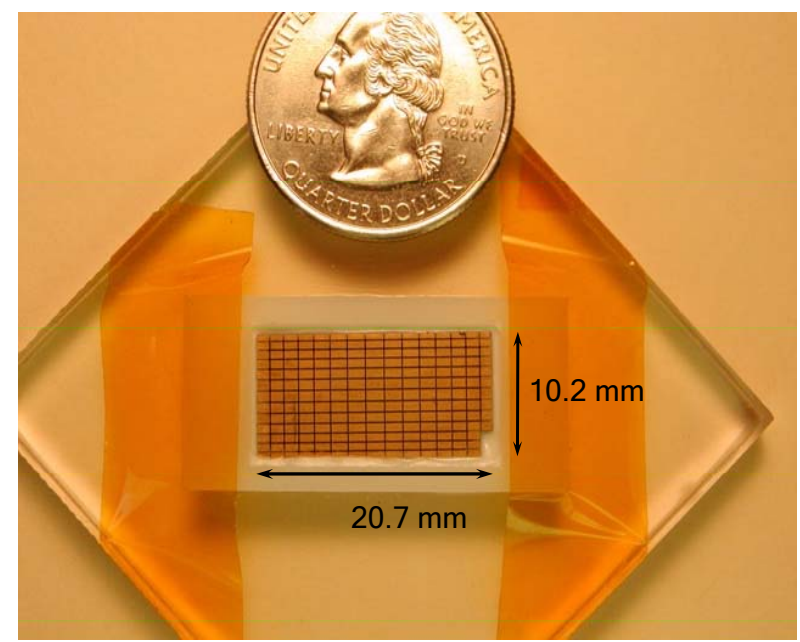

b

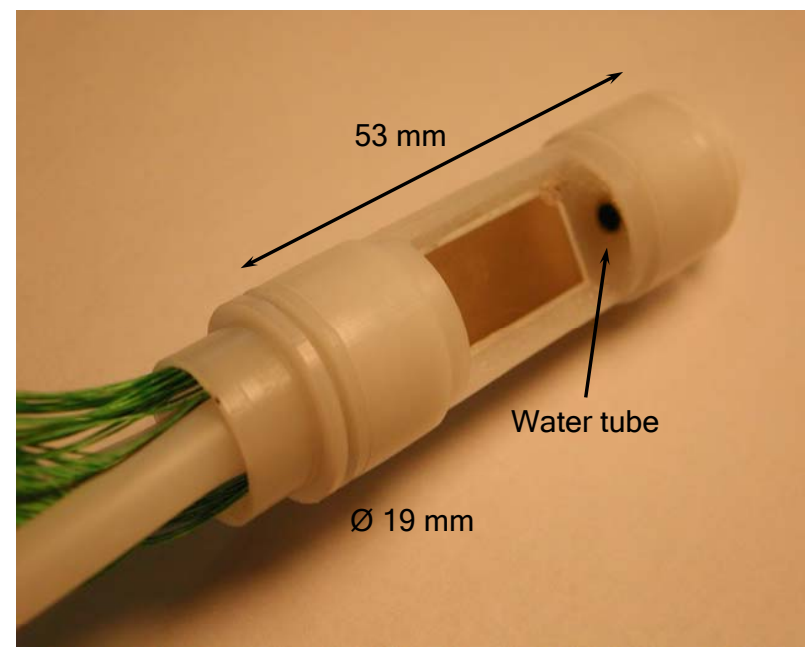

Fig. (6). (a) Photograph of the prototype array. Ceramic (PZT-8) diced into $15 \times 13$ elements, including 64 active elements. (b) Photograph of the array embedded in the housing.

Inc., Bloomfield, NY, USA), a $0.5 \mathrm{~mm}$ calibrated needletype PVDF hydrophone (SN: 679, Precision Acoustics Ltd., Dorchest, UK), and a digital oscilloscope (54622A, Agilent Technologies, Palo Alto, CA, USA). The array was submerged in an anechoic tank $\left(122 \times 51 \times 53 \mathrm{~cm}^{3}\right)$ containing degassed distilled water (room temperature, approximately $20^{\circ} \mathrm{C}$ ). To minimize cavitation, a customized degasser, built in-house, was utilized to reduce the dissolved oxygen content of the distilled water to 1-2 ppm. During all the exposimetry measurements for both on-axis and off-axis focusing, the level of the input driving power was carefully controlled to avoid damage to the hydrophone due to excessive high acoustic pressure. The input electrical power to the transducer elements for the exposimetry was $0.5 \mathrm{~W}$ per active element. The hydrophone was positioned at two preselected focal points in the acoustic field, $(0,0,30) \mathrm{mm}$ and $(0$, $-5,30) \mathrm{mm}$, from the geometrical center of the transmitter array. The pressure measurements were made in the $x$ direction (lateral) between -15 and $+15 \mathrm{~mm}$ (onedimensional, step size $=0.5 \mathrm{~mm}$ ) and in the $y z$-plane between -20 and $+20 \mathrm{~mm}$ of the $x$-direction and between 10 and $60 \mathrm{~mm}$ (two-dimensional, step size $=2.0 \mathrm{~mm}$ ) of the $z$ direction (axial).

\subsubsection{In Vitro Experimental System}

To determine the effectiveness of the array design for ultrasound thermal ablation, in vitro experiments were performed using fresh tissue. Porcine hearts were obtained from an abattoir on The Penn State University campus. The total time from death to experiments varied from 2 to 5 hours. The array and the fresh tissue were submerged in a water tank filled with degassed phosphate buffered saline (PBS) solution and the ultrasound beam from the array was positioned to sonicate perpendicularly to the surface of the tissue. The distance between the array and the surface of the tissue was $30 \mathrm{~mm}$. An ultrasound driving system was set to generate a continuous wave at $1.6 \mathrm{MHz}$ for all of the experiments. For the temperature rise estimation near the focal region of the tissue, temperature as a function of time was recorded using thermocouples via a data acquisition module (Model 8018, SuperLogics, Inc., Waltham, MA, USA) and an RS-232 to RS-485 converter. A thermocouple was implanted into the on-axis or off-axis focal zone below the surface of the tissue using a $16 \mathrm{G} 1 \frac{1}{2}$ needle (Precision Glide, Becton Dickinson and Co, Franklin Lakes, NJ, USA). The sonication duration for each case was 270 seconds and the corresponding temperature changes were measured during 300 seconds $($ step $=$ 10 seconds). Water temperature was maintained at $37 \pm 1^{\circ} \mathrm{C}$ using a circulator (Polystat ${ }^{\circledR}$ 12112-00, Cole Parmer, Vernon Hills, IL, USA). For the sonication, each active element of the sparse array was driven at an electrical power of $5 \mathrm{~W}$ for on- and off-axis focusing. The overall efficiency of the array, estimated based upon the exposimetry measurements, was about $40 \%$. After the sonication, the tissue was sliced with a scalpel and examined to assess lesion formation. Digital photographs and a ruler were used to record the results.

\section{RESULTS}

\subsection{Computational Simulations}

Simulation results of the ultrasound field of the normalized intensity calculated in the $x y$-plane from the each array model are shown in Fig. (7) for on-axis focusing at $\mathrm{A}:(0,0$, $30) \mathrm{mm}$, and for off-axis focusing at $\mathrm{B}:(0,-5,30)$ and $\mathrm{C}:(-5$, $0,30) \mathrm{mm}$, respectively. Intensity contours with levels between 0 and $-20 \mathrm{~dB}$ were plotted as indicated from the intensity color bar.

From the simulations, Fig. 7(a-c) show the calculation results of the sound field from the linear phased array. The results for on-axis focusing indicate that the suggested linear array achieved a focal zone length of about $20 \mathrm{~mm}$ at the aimed point $\mathrm{A}$, and the grating lobe level stayed below $-6 \mathrm{~dB}$ toward $\theta=21^{\circ}$ from the main lobe. For off-axis focusing at the point B, Fig. 7(b) shows that an unwanted focal zone caused by the grating lobe is formed below $-9 \mathrm{~dB}$ toward $\theta=32^{\circ}$ from the main lobe. The results seem to be acceptable for ultrasound ablation of the myocardium for both on-axis and off-axis focusing. However, the plot for off-axis focusing at the point $\mathrm{C}$ (Fig. 7(c)) shows that an unwanted focal 


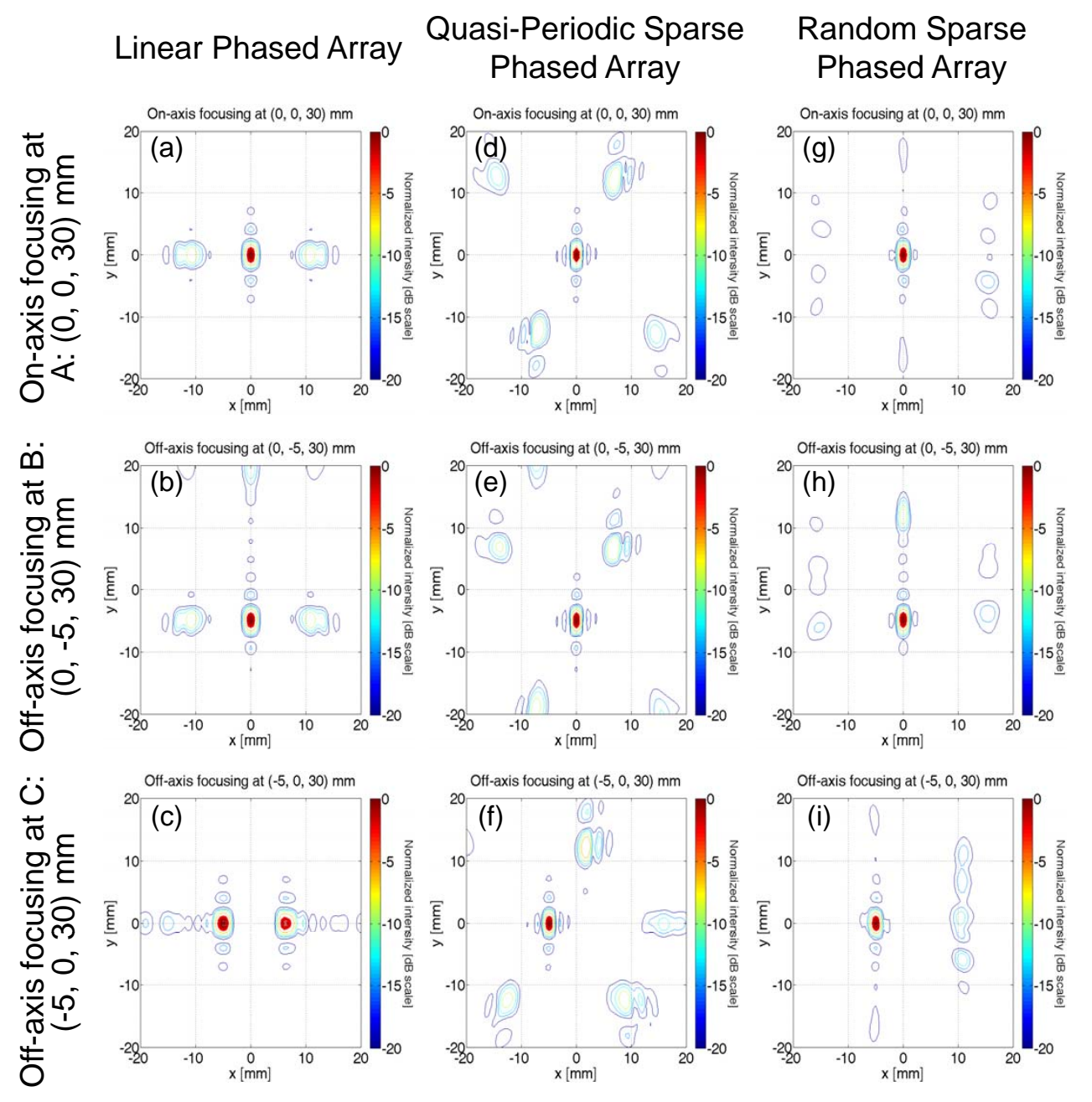

Fig. (7). Contours of the calculated ultrasound fields of the normalized intensity from the linear phased array, the quasi-periodic sparse phased array, and the random sparse phased array in the $x y$-plane, respectively for on-axis focusing at A: $(0,0,30) \mathrm{mm}$, off-axis focusing at $\mathrm{B}:(0,-5,30) \mathrm{mm}$, and off-axis focusing at $\mathrm{C}:(-5,0,30) \mathrm{mm}$, as indicated from the intensity color bar.

zone caused by the grating lobe is formed at $(6.5,0,30) \mathrm{mm}$ at a similar level of the main lobe. The grating lobe may have resulted from that the array geometries has a pitch of $2.5 \mathrm{~mm}$ in the $x$-direction and $1.2 \mathrm{~mm}$ in the $y$-direction, which is much larger than the $0.94 \mathrm{~mm}$ wavelength.

Fig. 7(d-f) show the simulation results of the sound field from the quasi-periodic sparse array. The designed sparse phased array achieved a beam width (FWHM) of about 2.5 $\mathrm{mm}$ at the aimed points, and the maximum steering angle extended toward $\theta= \pm 10^{\circ}$ from the main lobe. According to the simulation results, the sparse array model with tapered elements produced no significant grating lobes. Because equal element size arrays may introduce grating lobes in the beam pattern (results are not shown in this paper), an array with tapered elements was designed to significantly suppress grating lobes [28]. Since the array is quasi-periodic along a diagonal direction in the $x y$-plane, the plot in Fig. 7(f) shows grating lobes at $(2,12,30) \mathrm{mm}$. However, the results indicate that the grating lobe levels are at least $6 \mathrm{~dB}$ below the intensity at the focus; serious damage on normal tissue is not expected. In addition, a sparse array (64 active elements) can benefit from the reduced number of elements compared with a full matrix array (195 elements) [14, 29].
Simulation results of the sound field from the random sparse array are presented in Fig. 7(g)-(i). The results of both on- and off- axis focusing show that the random sparse phased array achieved good performances in focusing at the aimed points, as well as in depressing grating lobes although it has elements larger than one wavelength. For on-axis focusing, the well-confined focal zone ranges axially from 22 to $37 \mathrm{~mm}$ around the aimed point A. For off-axis focusing (Fig. 7(h) and (i)), the grating lobes are shown to be almost suppressed and kept below $-9 \mathrm{~dB}$.

\subsection{Exposimetry Tests}

Exposimetry results in Fig. (8) and Fig. (9) present the characteristics of the developed array. Figs. 8(a) and (b) compare the exposimetry measurements (the dashed line) and simulation (the solid line) of the sound field. For on- and off-axis focusing, the maximum normalized intensity differences between the measurements and the simulations were 10 and $-7 \mathrm{~dB}$, respectively. The calculated $-3 \mathrm{~dB}$ width of the main lobe was $\sim 2.7 \mathrm{~mm}$ for on-axis and $\sim 2.9 \mathrm{~mm}$ for offaxis steering. The averaged experimental results show agreement with the theoretical results $(n=4)$. The ultrasound fields of peak intensity for on-axis focusing at $(0,0,30) \mathrm{mm}$ 


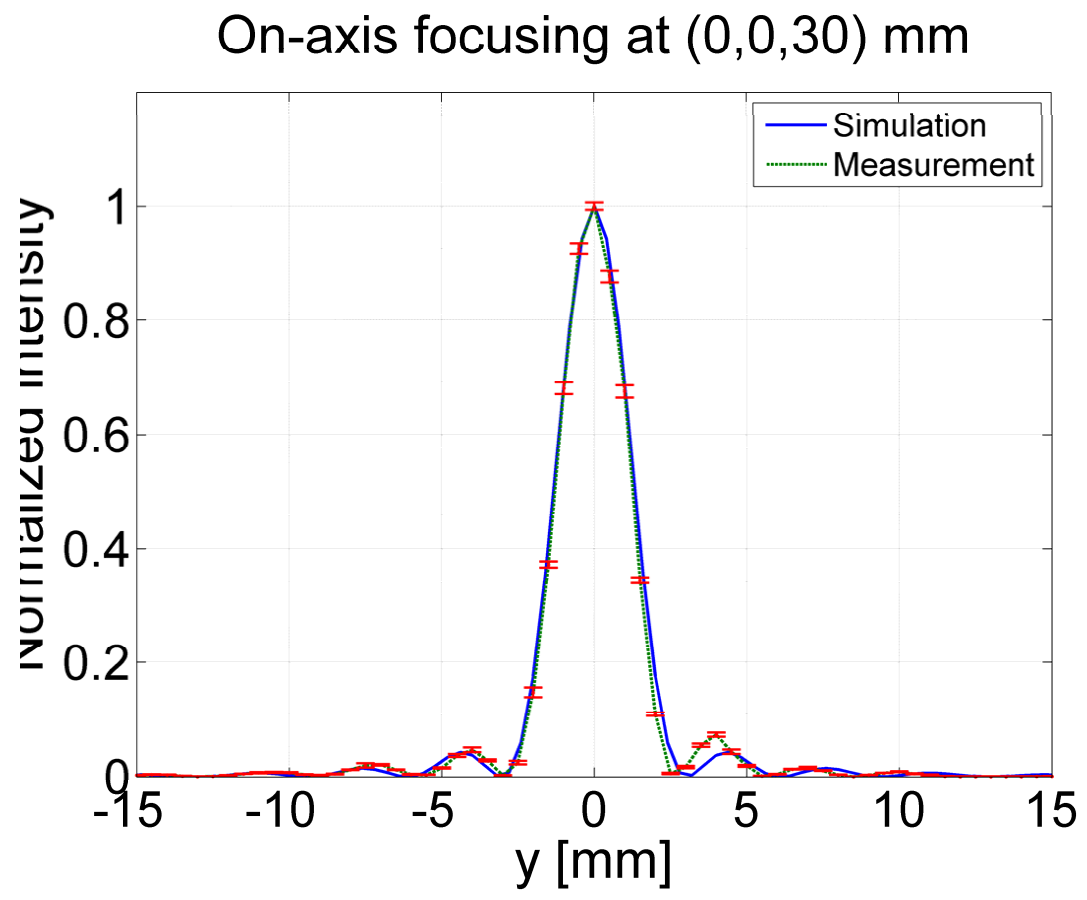

b

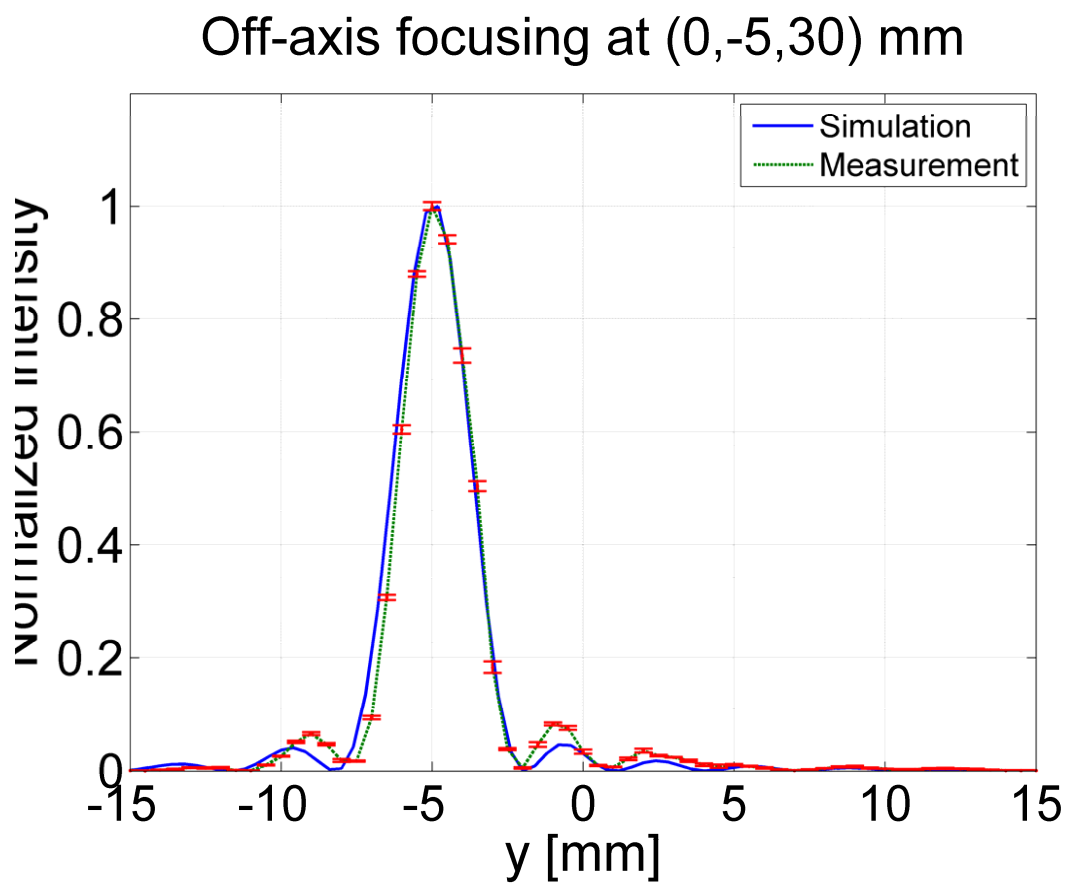

Fig. (8). Comparison of the results from simulation (solid line) and measurement ( $\mathrm{n}=4$, dashed line) for (a) on-axis at $(0,0,30) \mathrm{mm}$ and (b) off-axis focusing at $(0,-5,30) \mathrm{mm}$.

and off-axis focusing at $(0,-5,30) \mathrm{mm}$ with the prototype array show well-defined ultrasound focuses at the focal zones in Fig. 8(a) and (b), respectively. Precise on- and offaxis beam forming control was demonstrated without the significant grating lobes that may cause undesirable side effects of the treatment.

\subsection{In Vitro Experiments}

During the exposure period for in vitro thermal ablation, the temperature measured with the thermocouple in the focal zone rose from approximately 37 to over $50^{\circ} \mathrm{C}$, where appar- ent tissue damage near the surface of the tissue was observed by visual inspection within about 160 seconds. From the experiments, Fig. (10) shows an example of a typical ablation volume (on-axis focusing) created by the array in a porcine heart (large photograph, view in the $x z$-plane; small photograph, view in the $x y$-plane). Visual inspection was used as a method [30] to verify tissue damage and to distinguish the ablated volume on tissue samples. The border between the ablated tissue and the surrounding tissue can be readily identified in the photograph. The lesion in the tissue was $6 \times 5 \times 5 \mathrm{~mm}^{3}$ which was characteristic for the results. 

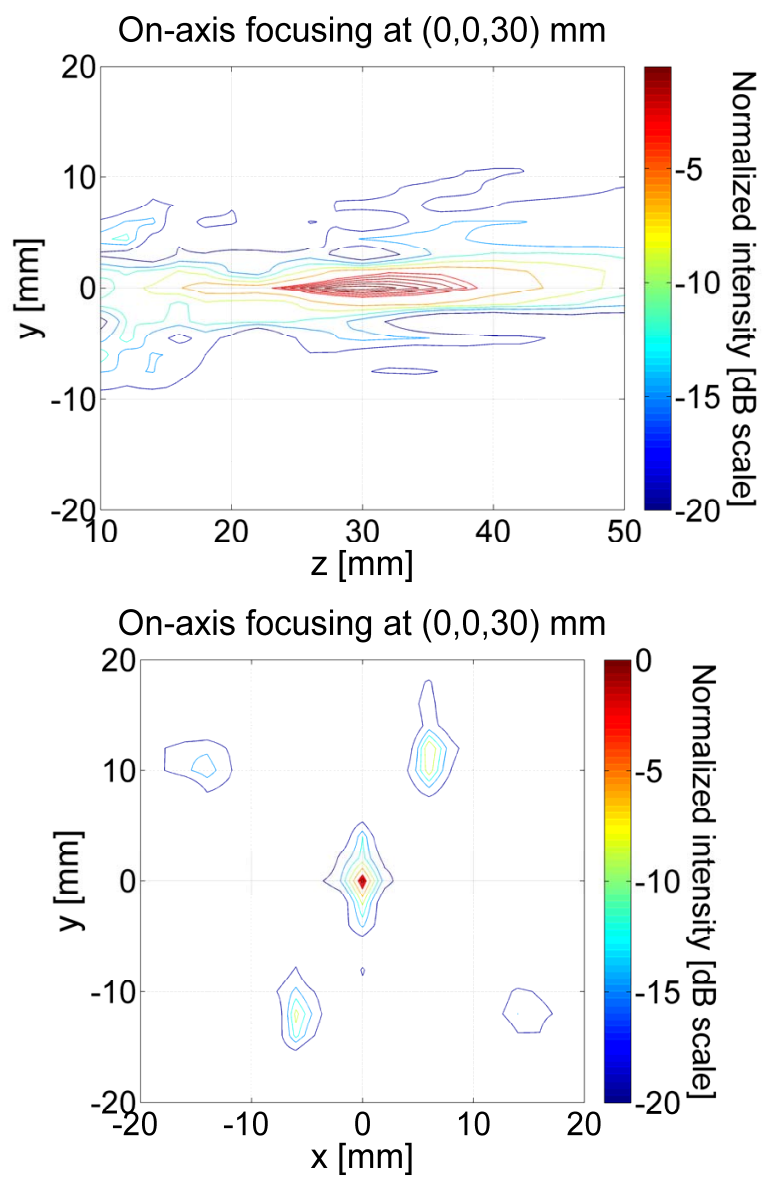

a

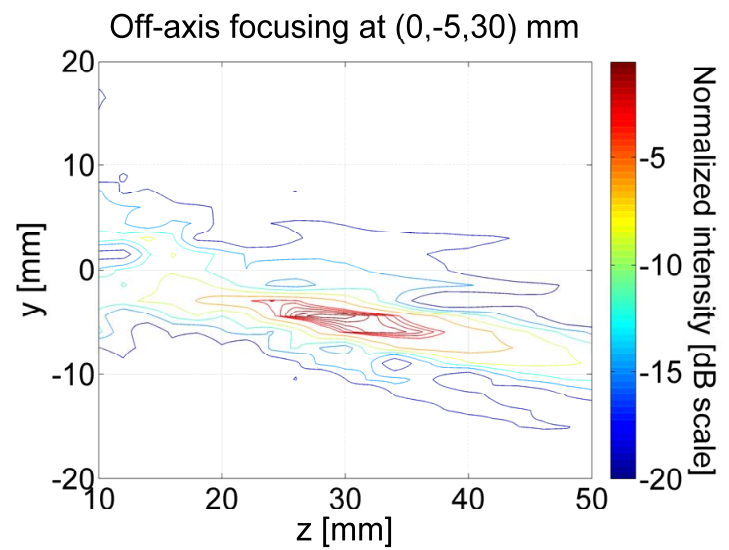

Off-axis focusing at $(0,-5,30) \mathrm{mm}$

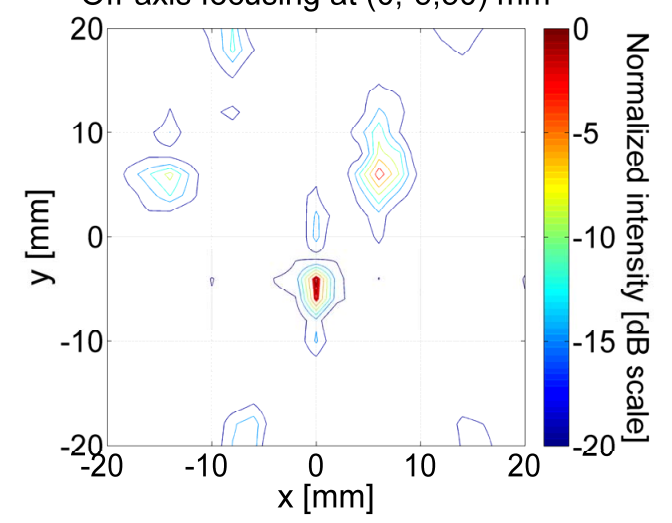

b

Fig. (9). Exposimetry measurement results. Normalized sound intensity contours in the $y z$-plane and the $x y$-plane, respectively for (a) on-axis focusing at A: $(0,0,30) \mathrm{mm}$, and (b) off-axis focusing at B: $(0,-5,30) \mathrm{mm}$, as indicated from the intensity color bar.

This indicates that the prototype array can deliver enough power (acoustic intensity of $300 \mathrm{~W} / \mathrm{cm}^{2}$ estimated based on the several exposimetry) to a focal point $30 \mathrm{~mm}$ deep inside the tissue to produce thermal lesions for cardiac ablation. However, a non-ablated area of tissue measuring $1.5 \mathrm{~mm}$ in diameter was observed within the lesion, seemingly due to an air gap located in front of this volume, which reflected and scattered the sound from its original path [16].

\section{DISCUSSION}

In this research the three different array geometries were characterized. Because the existence of the grating lobe may result in unwanted heating of the tissue with long exposures to ultrasound, the arrays should be designed to allow heat suppression. The large level of the grating lobe from the linear phased array is a direct result of violation of the phased array design criteria. In practice, the phased array should have a pitch size less than one half-wavelength to avoid raising significant grating lobes, especially when the beam is steered. For this reason, current research suggests designing the phased array with reduced pitch sizes but the same aperture sizes and the same number of transducer elements as the design of the quasi-periodic sparse array and the random sparse array. In the simulation results, the random sparse array showed excellence in both the focusing at the targeted area and controlling grating lobes in the calculated ultra- sound fields. The quasi-periodic sparse array, however, has the advantage of easy construction compared to the random sparse array even though it may generate weak grating lobes. The grating lobe problem is inevitable for a sparse array. The reason is that the sampling density of the sparse array is not sufficient to construct the pattern without aliasing. However, it is known that aperiodic array design can reduce the magnitude of grating lobes and improve the beamforming performance in the sparse array designs [14, 31].

This research has used a low electrical power of $5 \mathrm{~W}$ (maximum value of the amplifier for this research) per each active transducer element to drive the ultrasound applicator, which suffered from limitation of low temperature at focal zone (around $50^{\circ} \mathrm{C}$ ) with the consequence of long exposure time (270 seconds) to complete the thermal ablation. Together with a high-powered amplifier driving system, it will no doubt be advantageous to incorporate this ultrasound transducer in the higher speed treatment device for AF. In addition possible improvements concern the design of the ultrasound transducer. Although the suggested design of a sparse phased array provided thermal ablation, this design should benefit from optimization in the future.

As a continuation to the work described in this paper, an in vivo experiment is the imperative stage that should be involved. In general, in vitro experiments are carried out with 


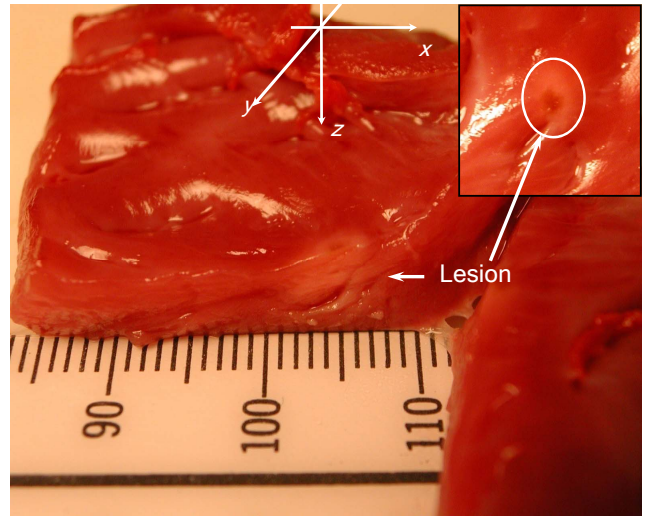

Fig. (10). Photographs of the results of in vitro experiment with excised porcine heart tissue (an example of on-axis focusing). A lesion and a non-ablated volume were created in the tissue (small photograph: view in the $x y$-plane).

excised fresh tissue samples. Thus, when testing the array for in vivo animal experiments under inhomogeneous and variable perfusion tissues, the exposure parameters for tissue ablation, such as sonication time and input driving power, should be modified. In addition, the isolated tissue samples are securely fixed at targeted position during in vitro experiments. In vivo experiments, on the other hand, will let the targeted area be free from a fixed position when the sonication starts due to the heart beating combined with respiration and animal movement during the ablation. Thus, an ultrasound energy delivery portfolio including real-time motion correction techniques with in vivo tests should be form the basis of follow up research.

Future studies would look at specific target locations and ablation. Visually there was no damage to adjacent structures; however, future studies are planned to evaluate gross and histological changes of the esophagus and other major anatomical structures of the thorax to ensure there is no subsequent damage.

Finally, living animal tests with image-guided surgery are strongly recommended. The precise determination of energy deposition and temperature in tissue volume during the thermal treatment is very important in positioning of the target points for ablations. Methods for monitoring temperature changes in in vitro experiments for this paper used thermocouples, which are a type of invasive probe. However, the process including invasive probes are not suitable for experiments with living animals. Techniques for temperature monitoring during hyperthermia have been developed during recent decades. Recent advances in non-invasive MR thermometry techniques have promoted imaging-guided thermal treatment. The technique uses the temperature dependence of the proton resonance frequency. Many studies have demonstrated the use of MR thermometry to successfully monitor ultrasound treatment [32-34]. MRI-guided surgery will promise precise location of the HIFU probe and in turn will ensure precise targeting of the desired area with quantitative temperature measurements.

\section{CONCLUSION}

In conclusion, the feasibility of a transesophageal ultrasound applicator for cardiac ablation was investigated to provide an effective means of focused ultrasound surgery having significant advantages over other treatment methods owing to its non-invasive nature. It was found that a twodimensional focused sparse phased array showed a potential application of ultrasound for transesophageal cardiac surgery in atrial fibrillation treatment. The experimental results indicate that the prototype array achieved the requirement for thermal ablation. For further development of this applicator, advanced research regarding the optimal design of the array design and high-performance ultrasound driving system, treatments under imaging guidance modality, and real-time motion correction techniques are recommended in future studies.

\section{ACKNOWLEDGEMENT}

This work was supported by Medtronic, Inc., Atrial Fibrillation Technologies, Minneapolis, MN, USA.

\section{REFERENCES}

[1] Thom T, Haase N, Rosamond W, et al. Heart disease and stroke statistics--2006 Update: a report from the American Heart Association Statistics Committee and Stroke Statistics Subcommittee. Circulation 2006; 113(6): e85-151.

[2] Haïssaguerre M, Jaïs P, Shah DC, et al. Spontaneous initiation of atrial fibrillation by ectopic beats originating in the pulmonary veins. N Engl J Med 1998; 339(10): 659-66.

[3] Benjamin EJ, Wolf PA, D'Agostino RB, Silbershatz H, Kannel WB, Levy D. Impact of atrial fibrillation on the risk of death: the Framingham Heart Study. Circulation 1998; 98(10): 946-52.

[4] Wolf P, Abbott R, Kannel W. Atrial fibrillation as an independent risk factor for stroke: the Framingham Study. Stroke 1991; 22(8): 983-88.

[5] Calkins H, Brugada J, Packer DL, et al. HRS/EHRA/ECAS Expert consensus statement on catheter and surgical ablation of atrial fibrillation: Recommendations for personnel, policy, procedures and follow-up. Heart Rhythm 2007; 4(6): 816-61.

[6] Jais P, Shah D, Hocini M, et al. Radiofrequency ablation for atrial fibrillation. Eur Heart J Suppl 2003; 5(suppl_H): H34-39.

[7] Khaykin Y, Morillo CA, Skanes AC, McCracken A, Humphries K, Kerr CR. Cost comparison of catheter ablation and medical therapy in atrial fibrillation. J Cardiovasc Electrophysiol 2007; 18(9): 90713.

[8] Cummings JE, Pacifico A, Drago JL, Kilicaslan F, Natale A. Alternative energy sources for the ablation of arrhythmias. Pacing Clin Electrophysiol 2005; 28(5): 434-43.

[9] Lynn JG, Zwemer RL, Chick AJ, Miller AE. A new method for the generation and use of focused ultrasound in experimental biology. $\mathrm{J}$ Gen Physiol 1942; 26(2): 179-93.

[10] Fry WJ, Mosberg WH, Barnard JW, Fry FJ. Production of focal destructive lesions in the central nervous system with ultrasound. $\mathbf{J}$ Neurosurg 1954; 11(5): 471-78.

[11] ter Haar G, Coussios C. High intensity focused ultrasound: physical principles and devices. Int J Hyperthermia 2007; 23(2): 89-104.

[12] Zimmer J, Hynynen K, He D, Marcus F. The feasibility of using ultrasound for cardiac ablation. IEEE Trans Biomed Eng 1995; 42(9): 891-97.

[13] Gentry KL, Smith SW. Integrated catheter for 3-D intracardiac echocardiography and ultrasound ablation. IEEE Trans Ultrason Ferroelectr Freq Control 2004; 51(7): 800-08.

[14] Goss S, Frizzell L, Kouzmanoff J, Barich J, Yang J. Sparse random ultrasound phased array for focal surgery. IEEE Trans Ultrason Ferroelectr Freq Control 1996; 43(6): 1111-21.

[15] Sanghvi N, Fry F, Zaitsev A, Olgin J. Cardiac ablation using high intensity focused ultrasound: a feasibility study. In: Proceedings of 1997 IEEE int Ultrasonics Symposium 1997; Vol. 2: pp. 1323-26.

[16] Smith NB, Hynynen K. The feasibility of using focused ultrasound for transmyocardial revascularization. Ultrasound Med Biol 1998; 24(7): 1045-54.

[17] Melodelima D, Salomir R, Chapelon J, Theillère Y, Moonen C, Cathignol D. Intraluminal high intensity ultrasound treatment in the 
esophagus under fast MR temperature mapping: in vivo studies. Magn Reson Med 2005; 54(4): 975-82.

[18] Yin X, Epstein LM, Hynynen K. Noninvasive transesophageal cardiac thermal ablation using a 2-D focused, ultrasound phased array: a simulation study. IEEE Trans Ultrason Ferroelectr Freq Control 2006; 53(6): 1138-49.

[19] Bonanomi G, Schwartzman D, Francischelli D, Hebsgaard K, Zenati MA. A new device for beating heart bipolar radiofrequency atrial ablation. J Thorac Cardiovas Surg 2003; 126(6): 1859-66.

[20] Lad VS, Patwardhan AM. Maze III replication using radiofrequency microbipolar coagulation. Heart Lung Circ 2004; 13(2): $139-44$.

[21] Cox JL, Schuessler RB, D'Agostino HJ, et al. The surgical treatment of atrial fibrillation. III. Development of a definitive surgical procedure. J Thorac Cardiovasc Surg 1991; 101(4): 569-83.

[22] Goodman J. Introduction to fourier optics. New York: McGrawHill Book Company 1968.

[23] Zemanek J. Beam behavior within the nearfield of a vibrating piston. J Acoust Soc Am 1971; 49(1B): 181-91.

[24] Tsao H, Wu M, Chern M, et al. Anatomic proximity of the esophagus to the coronary sinus: implication for catheter ablation within the coronary sinus. J Cardiovasc Electrophysiol 2006; 17(3): 26669.

[25] Oppenheim AV, Schafer RW, Buck JR. Discrete-time signal processing. $2^{\text {nd }}$ ed. USA: Prentice Hall; 1999.
[26] AIUM. Acoustic output labeling standard for diagnostic ultrasound equipment. American Institute of Ultrasound in Medicine 1998.

[27] IEEE. IEEE guide for medical ultrasound field parameter measurements. New York, NY: Institute of Electrical and Electronics Engineers 1990.

[28] Saleh K, Smith N. A 63 element 1.75 dimensional ultrasound phased array for the treatment of benign prostatic hyperplasia. BioMed Eng Online 2005; 4(1): 39.

[29] Holm S, Elgetun B. Properties of the beampattern of weight- and layout-optimized sparse arrays. IEEE Trans Ultrason Ferroelectr Freq Control 1997; 44(5): 983-91.

[30] Muratore R, Akabas T, Muratore IB. High-Intensity focused ultrasound ablation of ex vivo bovine achilles tendon. Ultrasound Med Biol 2008; 34(12): 2043-50.

[31] Hutchinson EB, Buchanan MT, Hynynen K. Design and optimization of an aperiodic ultrasound phased array for intracavitary prostate thermal therapies. Med Phys 1996; 23(5): 767-76.

[32] Chapman A, ter Haar G. Thermal ablation of uterine fibroids using MR-guided focused ultrasound-a truly non-invasive treatment modality. Eur Radiol 2007; 17(10): 2505-11.

[33] Furusawa $\mathrm{H}$, Namba $\mathrm{K}$, Nakahara $\mathrm{H}$, et al. The evolving nonsurgical ablation of breast cancer: MR Guided focused ultrasound (MRgFUS). Breast Cancer 2007; 14(1): 55-58.

[34] Smith NB, Buchanan MT, Hynynen K. Transrectal ultrasound applicator for prostate heating monitored using MRI thermometry. Int J Radiat Oncol Biol Phys 1999; 43(1): 217-25.

(C) Lee et al.; Licensee Bentham Open.

This is an open access article licensed under the terms of the Creative Commons Attribution Non-Commercial License (http://creativecommons.org/licenses/by-nc/3.0/) which permits unrestricted, non-commercial use, distribution and reproduction in any medium, provided the work is properly cited. 